\title{
El estudio de impacto ambiental como elemento de construcción de realidad. El caso de la central hidroeléctrica Porce III
}

The Environmental Impact Study as an Element of Reality Construction. The Case of Porce III Hydroelectric Station

O estudo de impacto ambiental como elemento de construção de realidade. O caso da central hidroelétrica Porce III

Gustavo Adolfo Muñoz Gaviria*

Recibido: 13 de febrero de 2018

Aprobado: 14 de octubre de 2018

Doi: http://dx.doi.org/10.12804/revistas.urosario.edu.co/territorios/a.6535

Para citar este artículo:

Muñoz Gaviria, G. A. (2019). El estudio de impacto ambiental como elemento de construcción de realidad. El caso de la central hidroeléctrica Porce III. Territorios, (41), 223-243. Doi: http://dx.doi.org/10.12804/ revistas.urosario.edu.co/territorios/a.6535

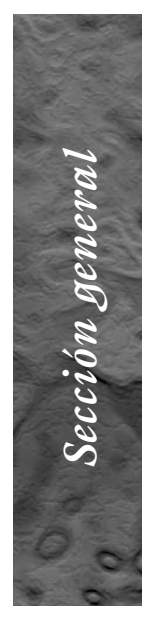

\footnotetext{
* Ingeniero Ambiental Universidad de Medellín, Especialista en Economía - Universidad de los Andes, Magíster en Gobierno - Universidad de Medellín, Doctor en Planeación Urbana y Regional - Universidad Federal de Rio de Janeiro. Docente de la Escuela Superior de Administración Pública-Territorial Antioquia-Chocó. Correo electrónico: gamgaviria@yahoo.es ORCID: bttp://orcid.org/00000001-6142-0605
} 
Palabras clave

Hidroeléctricas,

reasentamientos,

impactos sociales, gestión ambiental, desarrollo

territorial.

Keywords

Hydroelectric stations, resettlements, social impacts, environmental management, territorial development.

Palavras-chave

Hidroelétricas, reassentamentos, impactos sociais, gestão ambiental, desenvolvimento territorial.

\section{territarias 41}

\section{RESUMEN}

El presente trabajo aborda el estudio de impacto ambiental de la central hidroeléctrica Porce III, construida en la década del 2000 en Colombia desde una perspectiva sociológica, específicamente en relación al manejo de los efectos sociales. Se entiende esa "gestión de lo social" como un dispositivo de gobierno, un arreglo de líneas de visibilidad, enunciación, poder y subjetivación, por medio del cual se orienta la conducta de las poblaciones reasentadas. Se aborda particularmente el primer conjunto de líneas. Los datos provienen tanto del análisis documental, como de la experiencia de trabajo profesional del investigador en el reasentamiento estudiado. La información sobre normativa existente, justificaciones de la empresa y sus financiadores, estudios ambientales y medidas de manejo fue sometida a un análisis interpretativo a la luz de las categorías provistas por el referente teórico. El trabajo permite identificar la construcción de una realidad determinada, definida y delimitada por los instrumentos, discursos y técnicas del estudio de impacto ambiental. Establecida esa realidad, las empresas la gestionan al tiempo que hacen viable el negocio de generación de energía. Se concluye que otras realidades con mayores niveles de democratización pueden ser construidas para regular las interacciones entre comunidades, empresas y Estado en la definición de las formas de apropiación territorial.

\section{ABSTRACT}

The present work analyzes the environmental impact study of the Porce III hydroelectric power station, built in the 2000s in Colombia, from a sociological perspective, more specifically in relation to the social impacts" treatment. This "management of the social" is understood as a government apparatus: an arrangement of lines of visibility, enunciation, power, and subjectification by means of which the behavior of the resettled populations is oriented. The paper particularly approaches the first group of lines. The data came from both the documentary analysis and the professional work experience of the researcher in the studied resettlement. The researcher submitted the information on existing regulations, justifications of the company and its financiers, environmental studies and management measures to an interpretative analysis in light of the theoretical reference's categories. The study allows identifying that the construction, definition, and delimitation of a specific reality are brought about by means of instruments, discourses, and techniques of the environmental impact study. Once companies established that reality, they manage it while making viable the business of power generation. The research argues that other realities with higher levels of democratization can be constructed to regulate the interactions between communities, companies, and the State in defining forms of territorial appropriation.

\section{RESUMO}

O presente trabalho aborda o estudo de impacto ambiental da central hidroelétrica Porce III, construída na década de 2000, na Colômbia, desde uma perspectiva sociológica; mais especificamente, em relação à gestão dos efeitos sociais. Entende-se essa "gestão do social" como um dispositivo de governo, um arreglo de linhas de visibilidade, enunciação, poder e subjetivação, por meio do qual se orienta a conduta das populações reassentadas. Aborda-se especificamente o primeiro conjunto de linhas. Os dados provêm tanto da análise documental, quanto da 
experiência de trabalho profissional do pesquisador no reassentamento estudado. A informação sobre: normativa existente, justificações da empresa - e seus financiadores-, estudos ambientais e medidas de gestão, é submetida a análise interpretativa à luz das categorias fornecidas pelo referente teórico. O trabalho permite identificar a construção de uma realidade específica, definida e delimitada pelos instrumentos, discursos e técnicas do estudo de impacto ambiental. Estabelecida essa realidade, as empresas a gerem ao mesmo tempo que fazem viável o negócio de geração de energia. Conclui-se que outras realidades, com maiores níveis de democratização, podem ser construídas para regular as interações entre comunidades empresas e Estado na definição das formas de apropriação territorial.

\section{Introducción}

En el actual contexto de una eventual expansión del capital en Colombia hacia territorios que antes estaban bajo control de grupos armados ilegales, es de crucial importancia asegurar que aquellas poblaciones que fueron victimizadas por la violencia armada no lo sean nuevamente por los impactos y prácticas de los denominados proyectos de desarrollo. Como mecanismo para la regulación de estos proyectos, el Estado colombiano cuenta con un proceso de licenciamiento ambiental que ha visto en los últimos años intentos de las grandes empresas por limitarlo aún más tanto en términos de participación real de las comunidades afectadas en la definición del futuro de sus territorios, como de su capacidad de instrumento de comando y control. Se ha intentado hacer de este un proceso más simplificado, al punto de hablarse de un "licenciamiento exprés" (Medio Ambiente, 2014; Sleman-Chams \& Velásquez-Muñoz, 2016).

Los estudios de impacto ambiental son según la normativa vigente instrumentos necesarios para que las empresas accedan a las licencias y permisos de uso y explotación de recursos naturales, en consonancia son las directrices de la Ley 99 de 1993, a partir de la cual se modificaron los arreglos institucionales en materia de gestión ambiental en Colombia. Es común que estos estudios realicen la división de lo que se considera el medio ambiente entre “componentes" físico, biótico y social. En el caso colombiano, para la obtención de la licencia ambiental es necesario que quien la solicita realice una evaluación de los impactos que su proyecto producirá a cada uno de esos "componentes" y presente un plan para el manejo de dichos impactos.

En este trabajo se aborda lo que viene siendo llamado "Gestión Social" en proyectos de infraestructura: un conjunto de lógicas, prácticas, planes, programas y proyectos para el manejo de los impactos sociales con miras a dar cumplimiento a las licencias ambientales. Estudiamos en este artículo el caso de la central hidroeléctrica Porce III construida en AntioquiaColombia, en la década del 2000 por Empresas Públicas de Medellín (EPM). Nos enfocamos en el manejo dado al tema social de desplazamiento y reasentamiento. territarias 41

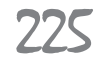


${ }^{1}$ Asumimos que los mineros constituian hogares unipersonales. En su página electrónica EPM afirma baber afectado aproximadamente 582 familias y 372 mineros: https://www.epm. com.co/site/Portals/0/medios_de_comunicacion/boletines_de_prensa/porceiii_ op_ccial.pdf

\section{territarias 41}

Pretendemos entender el efecto que los estudios de impacto tienen sobre la definición de los destinos de los territorios y las poblaciones afectados.

Cabe anotar que la central estudiada habría afectado un aproximado de 954 hogares entre familias y mineros ${ }^{1}$. De esta población, el $82 \%$ de los hogares fue indemnizado en dinero, mientras que solamente un $18 \%$ fue restituido con vivienda, proyecto económico (agropecuario o de comercio o servicios) y con acompañamiento por parte de la "Gestión Social".

Desde el punto de vista teórico, ampliamos aquí un trabajo anterior (Muñoz, 2016) en el que se abordó el conjunto de prácticas de la "Gestión Social" de proyectos hidroeléctricos como un dispositivo de gobierno en el sentido de Foucault (1980), Deleuze (1991) y Dean (2010) para el manejo de la conducta de los afectados. Este dispositivo de gobierno estaría inscrito en un proceso de mayor escala o una racionalidad mayor (Muñoz, 2016), que acompaña la etapa del capitalismo global y se correspondería con lo que Boltanski y Chiapello (2002) denominan "El nuevo espíritu del capitalismo", sobreviniente con el desmonte del Estado de Bienestar iniciado a finales del siglo Xx. Ese nuevo espíritu operaría paralelamente con lo que el mismo Boltanski (2013) denomina la forma de dominación gestionaria, en la que la instrumentalización de la ciencia económica y el perfeccionamiento de las técnicas del management y las herramientas de la gestión se imponen a la causalidad histórica en el desarrollo de narrativas amplias.

Según Foucault los dispositivos de gobierno estarían conformados por líneas de visibilidad, enunciación, poder y subjetivación en los que las líneas de visibilidad iluminarían el campo y los objetos a ser gobernados; las líneas de enunciación dotarían a una forma de gobierno de legitimidad y efecto de verdad al ser emanadas desde formas específicas de saber; las líneas de poder fijarían las posiciones de las personas y cosas para el ejercicio del poder a través de las técnicas e instrumentos, mientras que las líneas de subjetivación representarían la oposición o resistencia de los sujetos al dispositivo que los intenta gobernar.

En la lectura que Deleuze (1991) hace de Foucault, los dispositivos tienen como elemento esencial y primigenio las líneas de visibilidad pues estas hacen aparecer los objetos del dispositivo al conformarlos con las diversas formas que su luz proyecta. Sin embargo, no se trataría de que se iluminen objetos preexistentes, sino de un tipo de producción de formas de ser o estar. Según Dean (2010), dentro de los regímenes de gobierno, los objetos gobernados y las relaciones de autoridad y obediencia son definidos o iluminados por formas de visibilidad como diagramas, mapas, gráficos, etc. siempre a través de líneas de enunciación, con lenguajes y denominaciones provenientes de saberes específicos. Entendemos estas formas de dar visibilidad a los sujetos y objetos del 
dispositivo de la Gestión Social como un elemento en la construcción de la nueva realidad que se instaura después de la entrada de la empresa en el territorio para la construcción de la central hidroeléctrica.

Con el objeto de reflexionar sobre el carácter conflictivo de las interacciones entre empresas del sector, poblaciones y Estado y de entender las estrategias de los actores, intentamos hacer un puente entre el abordaje de gubernamentalidad de Foucault - y los desarrollos posteriores de Deleuze y Dean- con la sociología pragmática de la crítica de Luc Boltanski. Siguiendo a este último, proponemos entender la realidad como una construcción social (Boltanski, 2014) en la que juegan un papel fundamental las instituciones, al estabilizar temporalmente las posiciones de las personas y las cosas. Para este autor las instituciones tienen una contrapartida que él denomina la crítica como la oposición o resistencia al orden establecido por las instituciones, que les permite a los actores por ellas gobernados buscar mayores niveles de justicia o bienestar. Equiparamos entonces la "Gestión Social" al proceso de institución y pretendemos entender de qué herramientas se vale para construir el orden que pasa a ser la realidad para los actores.

Derivado de lo anterior, la hipótesis central de este trabajo es que los estudios de impacto ambiental, a partir de los cuales se formulan los planes de "gestión" de lo social, funcionan - junto con los anuncios del proyecto, los diagnósticos, las normas y los ejercicios de monitoreo-como líneas de visibilidad que iluminan un campo de afectaciones e intervenciones a ser posteriormente instituidas y contribuyen a la configuración de una realidad específica mediante la generación de efectos de verdad y jurisdicción - efectos que son centrales en la concepción de la relación entre derecho, verdad y poder en Foucault (1980)-

Pensamos de igual forma que el carácter "técnico" de los estudios, de las medidas y de los procedimientos mediante los que se implementan estaría facilitando la expansión del capital nacional y transnacional en el territorio colombiano, con perspectivas de aumentarse en los tiempos posteriores a las negociaciones con grupos armados de una forma que limita la garantía y expansión de los derechos de las poblaciones afectadas por este tipo de proyectos.

\section{Materiales y métodos}

Los resultados presentados y analizados en este trabajo hacen parte de una investigación de tipo cualitativo que desarrolló trabajo etnográfico con las familias y mineros impactados por el proyecto hidroeléctrico Porce III, así como análisis de los documentos corporativos de la empresa constructora, de sus consultores y de la normativa aplicable al reasentamiento de población.

Más específicamente, se presenta aquí una parte de los análisis documentales. La información sobre normativa existente, las justificaciones de la empresa y sus territarias 41 
consultores, los estudios ambientales y las medidas de manejo fue sometida a análisis interpretativo a la luz de las categorías provistas por el referente teórico para construir un objeto del conocimiento entendido como la realidad construida por las lineas de visibilidad del Dispositivo de la 'Gestión Social'.

\section{Resultados y discusión}

Los análisis de los discursos de la empresa, los financiadores, los consultores y los representantes del Estado contenidos en documentos oficiales permiten identificar algunos elementos como instrumentos, prácticas o lógicas para la conformación de una realidad sobre la cual actuar a través de planes, programas y proyectos para el manejo de las poblaciones afectadas por la central hidroeléctrica Porce III. Presentamos a continuación cinco elementos que configurarían un campo de visibilidad para la acción de esa "Gestión Social".

\section{Proyecto y cambio como creadores de realidad}

El primer argumento encargado de delimitar el tipo de realidad en el contexto de los proyectos hidroeléctricos es el que se encuentra en los documentos La Unidad de Planeación Minero Energética de Colombia (UPME), según el cual la "satisfacción de la demanda de energía del país" es una necesidad prioritaria. Allí los datos de demanda son estadísticamente relacionados con las mediciones de variación del producto interno bruto (PIB). La entidad llega a afirmar que "la demanda de energía eléctrica es uno de los mejores indicadores líderes de la actividad económica y, a su vez, el comportamiento de la demanda estaría estrechamente condicionado por el dinamismo de la actividad económica" (UPME, 2013, p. 4). Este argumento corresponde, sin embargo, a una visión cuestionable de desarrollo, en la cual este se confunde con el crecimiento económico, que tiene por objetivo principal aumentar el PIB sin importar la distribución del ingreso. Una completa crítica a esta visión se encuentra en Escobar $(2007,2012)$, quien explica la naturaleza "erosiva" y las razones de décadas de fracasos de esa visión de desarrollo impulsada para América Latina desde una perspectiva eurocéntrica y occidental.

Como representante de ese modelo de desarrollo, el proyecto hidroeléctrico con su "inexorabilidad" constituye el segundo "hecho" para la construcción de la nueva realidad. Como se evidencia en el documento de 2005 del Banco Interamericano de Desarrollo (BID), que financió parte de la central Porce III, este proyecto estaba en fase de estudios desde décadas atrás. En el mismo documento se defiende la pertinencia técnica del proyecto en los siguientes términos: "sus características [las de la central] están basadas en la optimización del potencial hidroeléctrico buscando el máximo aprovechamiento del recurso hídrico" (BID, 2005, p. 2), al emplear los volúmenes represados por otras tres represas aguas arriba. $\mathrm{Al}$ mismo tiempo, 
se defiende su viabilidad económica: "Porce III pertenece a la ruta de expansión de la generación de mínimo costo total" (BID, 2005, p. 2). Estas afirmaciones se suman al sentido común proveniente de ese modelo de desarrollo domínate que pregona la inexorabilidad de la expansión energética de un país "en vías de desarrollo", para justificar y tornar el proyecto en una realidad inminente. En tal contexto, cuando las poblaciones comenzaron a ser "consultadas" en la década del 90 (BID, 2005, Anexo 7), la realidad que estaban próximos a enfrentar ya había sido concebida diez años antes, en 1987, según el propio BID (2005, p. 17).

El mismo grado de inevitabilidad del proyecto fue expresado para los cambios sociales que sería necesario producir según sus promotores. Son múltiples las referencias en los discursos de la empresa y del BID a la necesidad de trasformar socialmente la región. En primer lugar, en los documentos de la empresa se establecía que las actividades tradicionales de la población a ser desplazada deberían ser sustituidas por otras. Como objetivos de corto plazo en el "Plan de Gestión Social", la empresa establecía:

Impulsar un proceso gradual de acercamiento a diferentes alternativas de vocación económica que permitan un cambio progresivo de actitud y mentalidad hacia proyectos económicos distintos a los tradicionales (...) Identificación y concertación con la comunidad al respecto de los proyectos económicos de carácter transitorio que permitan la adquisición de una nueva curva de aprendizaje (conocimientos y habilidades) al tiempo que se tornan en fuentes inmediatas de renta para las familias ${ }^{2}$ (EPM, 2002, p. 165 ).

Sin embargo, en el Estudio de Impacto Ambiental (EIA) no fueron explícitamente establecidas las razones que justificarían la necesidad del cambio que acabó por asumir un carácter de autoevidente.

La empresa también presentó, en anexo del documento del BID, una propuesta de contenido para el monitoreo de las familias reasentadas. Uno de los ítems del instrumento referente a "aspectos sobre relaciones sociales y culturales" analizaba la "actitud frente al cambio", con las siguientes posibilidades de evaluación: favorable, aceptación, ambivalencia, angustia o resistencia (BID, 2005, anexo 8 ); mientras que el "Cambio estructural de la población directamente impactada [...era caracterizado como un...] impacto negativo muy significativo"3 (BID, 2005, p.30). Como ejemplo final, en el mismo documento la empresa concluía como aprendizaje de la experiencia de la "Gestión Social" de la central Porce II, construida antes y aguas arriba de Porce III, lo siguiente:

en la esfera de lo cultural y psicológico se concluyó que es necesario posibilitar espacios para la elaboración del duelo del asentamiento abandonado y el bautizo del nuevo asentamiento, para proyectar un ser humano capaz de asumir cambios y
${ }^{2}$ Negrillas fuera del original.

${ }^{3}$ Negrilla fuera del original. 
${ }^{4}$ Negrilla fuera del original.

5 Negrilla fuera del original.

\section{territarias 41}

desafíos en la trasformación, construcción y adaptación a su nuevo entorno ${ }^{4}$ (BID, 2005, p. 19).

Queda ilustrado de esta forma cómo la empresa presentó, justificó y dio visibilidad a su proyecto como una realidad necesaria, inevitable y deseable para la sociedad como un todo y como generador de cambios inexorables para las poblaciones locales impactadas, las mismas que pasaría a denominar después como "poblaciones beneficiadas", cuando ellas se tornaran objeto de los programas de "Gestión Social".

\section{Efecto de institución}

El arreglo jurídico-legal desempeña un papel fundamental en la reproducción y estabilización de las relaciones de poder, así como lo hace la producción de "realidades" gobernables, como se analizó antes. La "Gestión Social" en la medida en que es determinada en medio de un conjunto de normas e instituciones (Muñoz, 2016) podría también ser leída como una "jurisdicción” (Foucault, 2008, p. 342 ) diseñada para "eliminar el hecho de la dominación y sus consecuencias" (Foucault, 1980, p. 95). El marco legal constituiría para nuestro caso el marco de referencia (Boltanski, 2013) de las medidas de la "Gestión Social" — si la entendemos como una práctica de la dominación gestionaria - donde la necesidad o el imperativo de sus medidas encuentra justificación. En otras palabras, la empresa no necesitaría de discursos ideológicos ni de rituales de valorización simbólica cuando ella esté actuando conforme a aquel marco técnico-normativo.

Para Foucault (1980) el derecho con sus leyes, dispositivos, instituciones y procedimientos pondría en funcionamiento relaciones de dominación que hacen ver el poder como legítimo de ser obedecido. Sin embargo, él también propone ver el derecho "no en términos de una legitimidad a ser establecida, sino, en términos de los métodos de subyugación que él

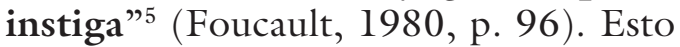
implicaría que sea metodológicamente necesario concentrarse en las formas del ejercicio del poder en sus extremidades, más que en el centro, para que sea posible captar esos métodos de subyugación en los puntos donde se torna "capilaridad (... en sus formas e instituciones más regionales y locales", para no correr el riesgo de intercambiar la cuestión de la dominación-subyugación por la de soberanía-obediencia (Foucault, 1980, p. 96). Siguiendo esa recomendación, se muestra a continuación un panorama del marco legal que encuadra la "Gestión Social" desde lo más general hasta lo más específico, que señala su relación con la función de creación de un campo de visibilidad, así como de enunciación de los objetos a gobernar.

Los "Planes de Gestión Social" constituyen la respuesta de las empresas a los requerimientos legales de manejo de impactos sociales de los proyectos. Este contexto normativo está determinado 

ambiente en el capitulo 3: derecho a un ambiente saludable, articulo 79; garantía estatal de desarrollo sostenible, prevención $y$ reparación de daños al

Licencias ambientales: Decreto 1220 , última vez modificado mediante decreto 1076 de 2015 ambiente, articulo $80 ; y$ acciones populares para la protección de los derechose intereses colectivos sobre el ambiente, articulo 88 .

Fuente: elaboración propia

fundamentalmente por lo que se denomina la Política Ambiental del país y más específicamente por el proceso de licenciamiento ambiental. En la figura 1 se muestra el engranaje normativo del cual hace parte la "Gestión Social" como práctica concreta de las empresas.

En la parte alta del engranaje se encuentra la Constitución Política que comparte principios ${ }^{6}$ con lo que es conocido y expresamente enunciado como Política Ambiental en Colombia. La definición más elaborada de esta última se encuentra en la Ley 99 de 1993, promulgada posteriormente a la Declaración de Río de Janeiro de junio de 1992 sobre Medio Ambiente y Desarrollo de la cual extrae sus princi-

pios. Además de establecer los principios de la política, la Ley 99 reestructuró la institucionalidad del país en materia de medio ambiente a través de la creación del Ministerio de Ambiente y el Sistema Nacional Ambiental.

La licencia ambiental en Colombia, así como en otros países de América Latina, es el instrumento que permite a las empresas el acceso al uso de los recursos y a la producción de impactos ambientales y sociales derivados de sus actividades. En Colombia se reglamentó la necesidad de solicitar licencias ambientales mediante el Decreto 1220 de 2005, modificado por el Decreto 1076 de 2015: tersitarias 41 231 
${ }^{7}$ Constitución Politica de Colombia: artículo 300; capitulo 2, sobre Planes de Desarrollo.

\section{territarios 41} 232
La licencia ambiental, es la autorización que otorga la autoridad ambiental competente para la ejecución de un proyecto, obra o actividad, que de acuerdo con la ley y los reglamentos pueda producir deterioro grave a los recursos naturales renovables o al medio ambiente o introducir modificaciones considerables o notorias al paisaje; la cual sujeta al beneficiario de esta, al cumplimiento de los requisitos, términos, condiciones y obligaciones que la misma establezca en relación con la prevención, mitigación, corrección, compensación y manejo de los efectos ambientales del proyecto, obra o actividad autorizada. La licencia ambiental llevará implícitos todos los permisos, autorizaciones y/o concesiones para el uso, aprovechamiento y/o afectación de los recursos naturales renovables, que sean necesarios para el desarrollo y operación del proyecto, obra o actividad. La licencia ambiental deberá obtenerse previamente a la iniciación del proyecto, obra o actividad (Ministerios de Ambiente y Desarrollo Sostenible, 2015).

Según la clasificación de Rodríguez y Espinoza (2002, p. 176), la licencia ambiental es un instrumento administrativo junto con otro tipo de permisos ambientales que serían también, según estos autores, instrumentos de gestión ambiental: los de regulación directa o comando y control, como normas de estricto cumplimiento; los instrumentos económicos, que operarían dentro de la lógica del mercado para orientar el comportamiento de los agentes, por ejemplo: las tasas por uso de recursos, y finalmente, una cuar- ta categoría de instrumentos incluiría la educación, la investigación y la asistencia técnica y la información ambiental. Sin embargo, Rodríguez y Espinoza (2002) también consideran la licencia ambiental como un instrumento de planeación, concepción que encontramos problemática pues asumirla de esa forma abriría la posibilidad al sector privado para ejercer una función que es prerrogativa y obligatoria del Estado colombiano ${ }^{7}$.

Un vistazo al surgimiento de los "estudios técnicos" como requisito para acceder al permiso de uso de los recursos muestra claramente que estos aparecieron con ese nombre en Colombia a partir de la entrada en vigencia del Código Nacional de Recursos Naturales Renovables y de Protección al Medio Ambiente, en el Decreto 2811 de 1974. Posteriormente, con la Ley 99 de 1993, se introdujo el término Estudio de Impacto Ambiental (EIA), el cual es definido en el artículo 57 como "información que deberá presentar ante la autoridad ambiental competente el peticionario de una licencia ambiental" (UPME, 1993). Esta definición fue refinada por sucesivos decretos, pero fundamentalmente se mantuvo que

El estudio de impacto ambiental es el instrumento básico para la toma de decisiones sobre los proyectos, obras o actividades que requieren licencia ambiental... Este estudio deberá corresponder en su contenido y profundidad a las características y entorno del proyecto, obra o actividad (Ministerio de Ambiente y Desarrollo Sostenible, 2015). 
Sin embargo, el EIA quedará más precisamente definido en relación a su contenido, este debe incluir: localización y descripción del proyecto; caracterización de los medios físico, biótico y socioeconómico; identificación, valoración y evaluación económica de los impactos sobre los diferentes elementos del territorio; plan de manejo de impactos con los respectivos proyectos y costos asociados; plan de monitoreo; plan de contingencias; plan de desmote y abandono, y plan de inversiones en los territorios impactados. Es este contenido el que le confiere al EIA su papel preponderante en la definición, delimitación y construcción de realidad pues configura un conjunto causalmente relacionado entre lo que se diagnostica como realidad y lo que se propone como herramienta para manejar-modificarla.

El Plan de Manejo Ambiental (PMA) es también requisito para obtener la licencia ambiental. Este es definido en la ley como

el conjunto detallado de actividades, que producto de una evaluación ambiental, están orientadas a prevenir, mitigar, corregir o compensar los impactos y efectos ambientales que se causen por el desarrollo de un proyecto, obra o actividad. Incluye los planes de seguimiento, monitoreo, contingencia, y abandono según la naturaleza del proyecto, obra o actividad (Ministerio de Ambiente y Desarrollo Sostenible, 2015, p. 1).

Este plan es en la práctica de la ejecución de los proyectos una especie de carta de navegación para quienes construyen los proyectos, pues contiene en detalle las medidas para el manejo de impactos con los cronogramas y costos; generalmente organizado por etapas del proyecto y discriminado por los componentes físico, biótico y social. Este es también la referencia de los entes de control y financiación para la fiscalización y el instrumento de la gestión socioambiental con que las empresas hacen el denominado "manejo integral de impactos" mediante medidas de prevención, mitigación, corrección y compensación (Ángel et al. citado en Díaz, 2010, p. 20). Aunque pueda ser entendido como un elemento para la visibilidad de objetos y sujetos de gobierno, consideramos que es el elemento por excelencia de las líneas de poder, es decir, el instrumento que condensa las técnicas del dispositivo como fue analizado por Muñoz (2017).

Finalmente, el Plan de Gestión Social aunque no figure específicamente en la normativa presentada antes, es la forma que toma la gestión de los aspectos sociales dentro del PMA.

\section{Los diagnósticos como construcciones}

Es una práctica común e incluso institucionalizada dentro del proceso de licenciamiento ambiental que los dueños de los proyectos hagan "diagnósticos" de la situación anterior a la construcción. En el caso de Porce III, el análisis de los discursos de la empresa y de los consultores permite identificar una estrategia de descalificación del estado de cosas territarias 41

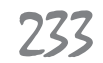


${ }^{8}$ Negrilla fuera del original.

\section{tersitarias 41}

antes del proyecto para promover la aceptación de este.

Tal es el caso de la empresa al referirse a las relaciones de la comunidad impactada como de "baja cohesión social" (EPM, 2002 , p. 160). Este enunciado es parte de la caracterización que la empresa hizo en el EIA para después proponer las medidas de manejo de los impactos sobre la población. Esa afirmación contrasta con la manifestación de uno de los mineros entrevistados en otra fase de la investigación, quien concluye al describir los lazos de solidaridad que funcionaban en su territorio antes de la llegada del proyecto que "el minero ve mucho las necesidades de los otros, hay solidaridad". Esta solidaridad se manifestaba no solamente en las transacciones de intercambio a crédito entre comerciantes y mineros, sino también en la práctica de compartir los espacios de extracción de oro con aquellos que lo necesitaran para cubrir necesidades puntuales como lo manifestaron otros mineros entrevistados por Muñoz (2016).

Una estrategia discursiva similar se observa en los consultores, especialistas en programas de "Gestión Social" de reasentamientos como ellos se autodefinen, al referirse a los cambios introducidos por el proyecto, el mismo año en que la construcción fue concluida:

Los atractivos futuros se van vislumbrando, comenzando por el hecho de tener una vía pavimentada con buenas especificaciones, y de la vista de la represa a lo largo de un buen recorrido, allí donde antes solamente era posible ver peñascos, donde apenas se adivinaban los meandros del río. Y la perspectiva turística que la oferta de nuevos paisajes para aquellos que visiten las montañas agrestes, que a pocas personas interesó en el pasado, irán dando, complementadas con la información histórica y técnica de lo que allí se produce para Colombia y el continente americano, la energía eléctrica, con tecnología del siglo $\mathrm{XXI}^{8}$ (Gómez, 2010. p. 185).

En este caso hay una combinación de afirmaciones que busca desvalorizar los atributos territoriales y las formas de vida de las poblaciones a ser manejadas, al mismo tiempo en que se presentan argumentos para sustentar la necesidad de la construcción del proyecto. Los consultores proponen la transformación de los territorios (des)calificándolos como "poco atractivos" y con relaciones sociales de producción "atrasadas".

Un ejemplo similar de este tipo de estrategia descalificadora se evidencia también en el caso brasileño de la promoción de la central hidroeléctrica Belo Monte, en Pará, estado del norte de Brasil. Siguiendo esa misma lógica, la Norte Energía, empresa dueña del proyecto afirma en la cartilla de divulgación sobre la central que:

La central Belo Monte llevará desarrollo a toda la región de su área de influencia, mejorando, en Altamira, la condición de vida de aproximadamente cinco mil familias que viven en palafitos y de decenas de familias de indígenas que viven en la ciudad, a merced

Gustavo Adolfo Muñoz Gaviria 
de los ciclos anuales de inundación, sin saneamiento básico ni otros servicios esenciales $^{9}$ (Norte Energía, 2011, p. 4).

De esta forma, la condición natural de variación de la hidrodinámica del río es mostrada como un infortunio para la población, desconociendo los efectos negativos de la estabilización de esos niveles que el proyecto provocaría en una parte del territorio (Muñoz, 2015). Una particularidad de esta central fue desviar el curso del río y causar desecamiento en una importante porción del territorio, interrumpiendo la dinámica inundaciónsequía aprovechada por la población para la diversificación productiva: agricultura en periodos secos y pesca en períodos de inundación.

Este tipo de estrategia descalificadora con fines de producción de objetos y sujetos gobernables ha sido documentado por algunos autores, entre ellos Escobar (1986) y Ferguson y Lohmann (1994). El primero describe la descalificaciónproducción hecha por los países industrializados de un "tercer mundo" sobre el cual implementar los planes para llevarlos por la senda del "desarrollo", convirtiéndolos en espacios gobernables a través de múltiples "reformas estructurales". De forma similar, los segundos detallan la construcción contraevidente de Lesotho en África, como un lugar atrasado y ávido de recibir las intervenciones de los programas de desarrollo rural ofrecidos y financiados por los organismos multilaterales.

\section{"Impacto" e "impactado" como representaciones de una realidad}

El impacto, en el lenguaje utilizado en el campo de la gestión y más específicamente en la "gestión socioambiental", es definido según la Organización Internacional para la Normalización (ISO, en inglés) como un cambio en el medio que rodea a una organización, como consecuencia del ejercicio de sus actividades, que puede ser positivo o negativo (Icontec, 2015). En la misma línea se encuentra la definición proveniente de las ciencias administrativas del sector privado según la cual "Los impactos socio-ambientales son el objeto de la gestión socio-ambiental y pueden ser manejados de tres formas: prevención, mitigación o compensación" (Ángel et al., 2001 citado en Díaz, 2010, p. 20). Como se vio en la sección anterior, esta definición es retomada e incorporada en la estructura legal del país, lo que le confiere la fuerza simbólica propia de su inclusión en el lenguaje estatal (Bourdieu, 2007).

El impacto como nueva realidad creada por el proyecto es manejado conceptual e instrumentalmente a través del EIA. Cada impacto es identificado, definido y delimitado por las empresas que establece así un espacio de acción para sus planes de gestión y produce una "definición selectiva" de los impactos sociales, como también lo documenta Muñoz (2013) para el caso de la industria minera en la Amazonía brasileña.

Una primera delimitación de los objetos y sujetos impactados opera a través
${ }^{9}$ Negrilla fuera del original. tersitarios 41

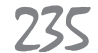


de una identificación geográfica de los efectos del proyecto al ser definidas las "áreas de influencia directa" en los diferentes municipios afectados, en el EIA. En la “caracterización” poblacional que la empresa hace, la distribución geográfica es representada en tablas como la que se muestra a continuación (tabla 1 ). Los te- rrenos y viviendas impactadas presentadas en esta pertenecen a las veredas definidas como de "influencia directa" en los diferentes municipios. Una representación geográfica fue hecha también sobre mapas digitalizados del territorio (a los cuales esta investigación no tuvo acceso), los mismos que constituyeron un elemento

Tabla 1. Identificación-distribución geográfica de los terrenos y viviendas impactados

\begin{tabular}{|c|c|c|c|c|c|c|c|}
\hline & \multicolumn{3}{|c|}{ Predios } & \multicolumn{4}{|c|}{ Viviendas } \\
\hline Municipio & Veredas & Total & Con título & Poseedores & Total & Convencional & No convencional \\
\hline \multirow{5}{*}{ Amalfi } & El Guaico & 1 & 1 & 0 & 3 & 0 & 3 \\
\hline & La Cristalina & 1 & 1 & 0 & 6 & 3 & 3 \\
\hline & La Manguita & 6 & 66 & 0 & 35 & 1 & 34 \\
\hline & Mangos & 1 & 1 & 0 & 3 & 0 & 3 \\
\hline & María Teresa & 6 & 6 & 0 & 10 & 4 & 6 \\
\hline \multirow{5}{*}{ Anorí } & Pajonal & 6 & 5 & 1 & 22 & 17 & 5 \\
\hline & El Limón & 15 & 15 & 0 & 59 & 25 & 34 \\
\hline & El Retiro & 11 & 10 & 1 & 29 & 0 & 29 \\
\hline & El Roble & 5 & 5 & 0 & 1 & 0 & 1 \\
\hline & El Zafiro & 6 & 6 & 0 & 17 & 0 & 17 \\
\hline \multirow{3}{*}{ Guadalupe } & Bramadora & 5 & 4 & 1 & 183 & 57 & 126 \\
\hline & Plan de Pérez & 12 & 12 & 0 & 11 & 7 & 4 \\
\hline & Puente Acacias & 3 & 3 & 0 & 83 & 25 & 58 \\
\hline Gómez Plata & El Oso & 1 & 1 & 0 & 0 & 0 & 0 \\
\hline Sin datos & & 2 & 2 & 0 & 0 & 0 & 0 \\
\hline \multicolumn{2}{|l|}{ Total } & 81 & 78 & 3 & 462 & 139 & 323 \\
\hline \multicolumn{2}{|l|}{$\%$} & & 96 & 4 & & 30 & 70 \\
\hline
\end{tabular}


de visibilización de los sujetos en el territorio para el desarrollo de las actividades de "Gestión Social".

La información recolectada en los censos de población fue también usada para identificar, cuantificar y clasificar a los sujetos impactados. En la tabla 2 se expone un ejemplo de la representación de una parte de esa información. Este caso muestra una clasificación múltiple que agrupa los sujetos: (a) según presenten agregación familiar o no; (b) según su relación de propiedad con la tierra o vivienda, y (c) según su actividad económica.

Tabla 2. Identificación-clasificación de la población impactada

\begin{tabular}{|c|c|c|c|}
\hline \multirow{2}{*}{ Tipo de población } & \multicolumn{2}{|c|}{ Población } & \multirow{2}{*}{ Composición } \\
\hline & No. Familias & No. Personas & \\
\hline $\begin{array}{l}\text { 1. Propietarios de } \\
\text { predios hasta } 5.0 \text { ha }\end{array}$ & 44 & 183 & $\begin{array}{l}10 \text { familias de mineros de oro de veta } \\
4 \text { familias de mineros de oro de aluvión } \\
3 \text { familias de mineros de veta y aluvión } \\
1 \text { familia de jornaleros agropecuarios } \\
19 \text { familias de pequeños productores } \\
\text { agropecuarios } \\
7 \text { familias de comerciantes (poseedores de negocios) }\end{array}$ \\
\hline $\begin{array}{l}\text { 2. Propietarios de } \\
\text { predios mayores de } \\
5.0 \text { ha }\end{array}$ & 10 & 42 & $\begin{array}{l}2 \text { familias de mineros de aluvión } \\
8 \text { familias de productores agropecuarios }\end{array}$ \\
\hline $\begin{array}{l}\text { 3. Arrendatarios de } \\
\text { viviendas }\end{array}$ & 18 & 75 & $\begin{array}{l}5 \text { familias de mineros de oro de veta } \\
7 \text { familias de empleados de empresas } \\
3 \text { familias de mineros de oro de aluvión } \\
1 \text { familia de jornaleros agropecuarios } \\
2 \text { familias de comerciantes (poseedores de } \\
\text { negocios) }\end{array}$ \\
\hline $\begin{array}{l}\text { 4. Poseedores de } \\
\text { predios por ocupación }\end{array}$ & 25 & 104 & $\begin{array}{l}13 \text { familias de mineros de oro de veta } \\
2 \text { familias de empleados de empresas } \\
5 \text { familias de mineros de oro de aluvión } \\
3 \text { familias de jornaleros agropecuarios } \\
2 \text { familias de mineros de veta y aluvión }\end{array}$ \\
\hline $\begin{array}{l}\text { 5. Habitantes de } \\
\text { "cambuches" }\end{array}$ & 6 & 25 & 6 familias de mineros de oro de aluvión \\
\hline $\begin{array}{l}\text { 6. Aparceros y } \\
\text { vivientes }\end{array}$ & 10 & 42 & $\begin{array}{l}1 \text { familia de aparceros, productores agrícolas } \\
9 \text { familias de administradores, cuidanderos y } \\
\text { vaqueros en fincas de producción agropecuaria }\end{array}$ \\
\hline Total & 113 & 471 & \\
\hline
\end{tabular}

Fuente: EPM (2002). 
La tabla anterior es un ejemplo de la caracterización inicial que la empresa hizo de la población en la fase de estudios. Según el EIA (EPM, 2002), una amplia lista de ítems es chequeada para la formulación del PGS: tamaño y distribución de la población; acceso, uso y disposición de recursos productivos; tipo de relación establecida con el medio; estrategias adaptativas y diversidad cultural; formas de poder y niveles de cohesión, organización y participación, y disponibilidad, cobertura y calidad de los servicios públicos básicos (acueducto, alcantarillados, atención en salud, educación, vivienda, empleo, ocio, etc.).

Por su parte, los impactos del proyecto son presentados también en tablas en las que son nombrados, clasificados en "positivos" o "negativos", "directos" o “indirectos", y finalmente, se les asigna una "evaluación de magnitud". Un ejemplo de esta forma de mostrar y clasificar los cambios e impactos inducidos por el proyecto se muestra en la tabla 3. Esta corresponde a una simplificación de lo que se conoce como la matriz de impacto ambiental, que es elaborada por las empresas e incluida en el EIA. Ella contiene también una columna con el programa que estaría encargado de la gestión del impacto.

Este tratamiento de las afectaciones a personas, poblaciones y territorios al ser una forma de representación hace visibles unas de ellas e (in)visibiliza otras. Por esa razón, la potestad de la denominación de las afectaciones y la definición de las medidas para su manejo confiere beneficios en la confrontación de intereses o visiones

Tabla 3. Síntesis de impactos socioambientales del proyecto y programas de gestión

\section{territarios 41}

\begin{tabular}{|l|c|c|c|c|c|c|}
\hline \multicolumn{1}{|c|}{ Impacto } & Positivo & Negativo & Directo & Indirecto & Calificación & $\begin{array}{r}\text { Programa de gestión } \\
\text { socioambiental }\end{array}$ \\
\hline $\begin{array}{l}\text { Cambio } \\
\text { estructural de } \\
\text { la población } \\
\text { afectada } \\
\text { directamente }\end{array}$ & 1. Etapa de construcción del proyecto (obras civiles y líneas de transmisión)
\end{tabular}

Fuente: BID (2005). 
del mundo. Se trata del ejercicio de la fuerza del capital simbólico proveniente del uso y dominio de las categorías que logran imponerse en la denominación de objetos, sujetos y relaciones. Un ejemplo de esta disputa simbólica, en este caso por la definición de lo que se entendía por "impactado" en la lucha del Movimiento de los Impactados por Represas (MAB, en portugués), es documentado por Vainer (2008), quien muestra cómo las reivindicaciones y el reconocimiento de derechos de los afectados ha dependido de lo que se ha entendido por "impactado" en diferentes momentos y lugares de esa lucha, convirtiéndola en una por la definición de ese concepto, y cómo esas reivindicaciones han alcanzado reconocimiento y fuerza simbólica mediante diversas estrategias del MAB.

\section{El "monitoreo" como forma de ver y controlar}

De forma concomitante con la implementación del Plan de Gestión Social se desarrolló en Porce III un proceso que contribuyó a mantener la visibilidad de los objetos y sujetos para gobernarlos desde la óptica de la técnica, se trata del denominado "Plan de Monitoreo".

Este plan es parte del EIA y el instrumento que permite a la empresa hacer seguimiento y vigilar la adopción de las recomendaciones hechas por los funcionarios de la "Gestión Social" en materia económica, social y ambiental a las familias reasentadas. La idea subyacente al monito- reo era la de supervisar el "desempeño" de las familias en cada uno de los ámbitos de su adaptación al nuevo entorno y nuevas formas de producción y vida.

Para llevar a cabo el monitoreo, la "Gestión Social" de Porce III definió unos "índices de desempeño" que evaluaban diversas variables en cada ámbito. Estos índices se medían semestralmente y se reportaban en gráficos de desempeño que eran presentados a las familias, lo que acababa por incorporarlas en la lógica del "mejoramiento del desempeño" en las tareas de manejo de recursos económicos, de conservación ambiental o de asociación comunitaria. Los consultores son enfáticos en la conveniencia de que

el sistema de monitoreo y evaluación sea participativo, es decir, que se diseñen mecanismos para garantizar que los sujetos de intervención en los diferentes niveles del plan de gestión socio-ambiental participen en la medición de los resultados, efectos e impactos (Díaz, 2010, p. 188).

El involucramiento de las familias por iniciativa de la empresa para participar en el proceso de evaluación de desempeño habría permitido a la empresa el gobierno a distancia (Miller \& Rose, 2012) de estas, una característica que estos autores atribuyen a las formas liberales avanzadas de gobierno en las cuales la invitación al autocontrol basado en cifras, cálculos y mediciones es una característica central.

Adicionalmente, los resultados del análisis periódico del "desempeño" de territarios 41 239 
las familias en los diversos ámbitos del reasentamiento eran usados también para el reporte de los avances en la gestión que los consultores u operadores hacían a la empresa y a su vez para que la empresa los presentara a las autoridades ambientales y a los entes financiadores.

\section{Conclusiones y recomendaciones}

El análisis anterior permitió una aproximación al entendimiento de algunas prácticas, instrumentos y discursos de la lógica de los EIA como elementos que contribuyeron a definir el campo de visibilidad de la "Gestión Social" en tanto dispositivo para el gobierno de las poblaciones reasentadas por el proyecto Porce III.

Según lo visto, el sistema de visibilidad y observación de la "Gestión Social” como dispositivo de gobierno se caracterizaría por (a) convertir el interés particular de una empresa en un cambio necesario, inevitable y deseable para el territorio a impactar; (b) hacer un diagnóstico descalificador del territorio y de la población a impactar para sobre este definir una línea de intervención; (c) aprovechar el beneficio simbólico que confiere la denominación estatal a través del andamiaje normativo a las afectaciones generadas y a sus formas de manejo; (d) hacer de la noción de impacto y su forma de definirlo, delimitarlo y manejarlo su ámbito de acción, y (e) establecer una forma de observar el comportamiento de los reasentados y de gobernarlos a distancia por medio del "monitoreo de su desempeño".
Este campo de visibilidad constituiría un "diagrama de poder" (Dean, 2010) o una "máquina óptica para ver sin ser visto" como lo expuso Deleuze (1991) al respecto de los dispositivos, profundizando en la idea del panóptico de Bentham desarrollada por Foucault (1980) con el objetivo de entender la cuestión de la visibilidad de los cuerpos, individuos y cosas en un sistema centralizado de observación.

La posibilidad de dar visibilidad a las poblaciones impactadas por medio de este manejo "técnico" de los impactos para después gestionarlos a través de los planes, los programas, los proyectos y las actividades de la "Gestión Social" establecería la relación que en palabras de Latour se da entre "el ver y el hacer": una relación que permitiría "pensar con ojos y manos" dentro de un dispositivo de gobierno (citado en Dean 2010, p. 41). El campo de lo visible creado por las normas, los instrumentos y el lenguaje de la empresa y el Estado dentro de la acción del dispositivo de la "Gestión Social" configuran así una realidad que contiene en ella misma lo que es posible y legítimo hacer, una realidad “maleable a la intervención" (Miller \& Rose, 2012, p. 26).

Sin embargo, como se viene afirmado en este trabajo, se trata de una realidad construida, un recorte del universo de lo posible o de lo que Boltanski (2013) denomina el mundo, es decir, "todo lo que pasa" (p. 444), que a su vez constituye la fuente de las críticas a la realidad institucionalizada que configura los procesos de dominación. En mundos diferentes 
o incluso mundos de la tradición de los pueblos, se encontrarían otras lógicas de acción, otras racionalidades para la disposición de los objetos y las cosas, es decir, otros órdenes sociales; lo que siempre abre espacio para buscar mayores niveles de justicia tanto en los medios (normas, instrumentos, procedimientos, etc.), como en los fines (básicamente representados en visiones o ideales de sociedad).

Es en el mundo donde tendrían que buscarse elementos para la construcción de otras realidades desde formas diferentes de concebir cuestiones como la política energética del país, hasta la revisión de cuestiones relativas a la preservación de los derechos de las poblaciones locales sobre el territorio y sus recursos, pasando por la modificación de las normas y el propio proceso de reasentamiento de poblaciones, de forma tal que las interacciones entre empresas, comunidades y Estado estén mejor balanceadas en términos de capacidad de defensa de los intereses de los afectados y en general todas aquellas modificaciones que las poblaciones organizadas defiendan como reivindicaciones en los contextos de disputas referentes a la construcción de proyectos de infraestructura.

Como se mostró en este trabajo, la construcción de otras realidades implica crear otras visibilidades, otras formas de entender las cuestiones relativas al desarrollo, al futuro de los territorios y a la reproducción sociocultural de las poblaciones que tradicionalmente han adquirido el derecho a permanecer y a mejorar sus condiciones de vida. Implica incluir de forma real otras voces, modificar la concepción de lo que es ser "impactado", modificar la institucionalidad para que se garantice su inclusión como voces decisorias tanto en lo tocante al desarrollo deseado para los territorios, como a los procedimientos de reasentamiento cuando este sea acordado.

En este último caso sería necesario garantizar procesos colectivamente construidos y acordados tales como programas de desarrollo local, proyectos productivos, "contratos de reasentamiento" o su equivalente, etc., usando instrumentos igualmente más participativos y democráticos como la cartografía social o mapeo participativo para la "producción de sentidos y territorios" (Acselrad, 2013, p. 5), de modo tal que los beneficios simbólicos del uso de los instrumentos no sean, por ser las que los conciben, exclusivamente para las empresas. Consideramos también fundamental la existencia de las mediaciones que las comunidades afectadas decidan incluir para su interacción con las empresas en los espacios decisorios. Estos mediadores pueden ser aliados estratégicos de las comunidades provenientes de redes de movimientos sociales o de instituciones que se identifiquen con sus reivindicaciones.

La democratización de la concepción de los instrumentos será clave para contribuir a devolverle a la definición del tipo de desarrollo deseado su carácter político ante la expansión de la racionalidad gestionaria y el imperio de la técnica difundido por medio de dispositivos como el de la "Gestión Social" analizado en este trabajo, territarias 41 241 
que acaba despolitizando las interacciones sociales en esos contextos.

\section{Referencias}

Acselrad, H. (Org.) (2013). Cartografia Social, terra e território. Río de Janeiro, Brasil: Universidad Federal de Río de Janeiro - Instituto de Investigación y Planeación Urbana y Regional (IPPUR).

Banco Interamericano de Desarrollo (BID). (2005). Proyecto Central Hidroeléctrica Porce III (CO-L1005).

Boltanski, L. (2013). Sociologia da crítica, instituições e o novo modo de dominação gestionária. Sociologia e Antropologia, 3(6), 441-463.

Boltanski, L. (2014). De la crítica. Compendio de sociología de la emancipación. Madrid: Akal.

Boltanski, L., \& Chiapello, E. (2002). El nuevo espiritu del capitalismo. Madrid: Akal.

Bourdieu, P. (2007). Razones prácticas. Sobre la teoría de la acción. Barcelona: Anagrama.

Colombia, Congreso de la República. Ley 99 de 1993. Por la cual se crea el Ministerio del Medio Ambiente, se reordena el Sector Público encargado de la gestión y conservación del medio ambiente y los recursos naturales renovables, se organiza el Sistema Nacional Ambiental (SINA) y se dictan otras disposiciones (22 diciembre 1993).

Colombia, Ministerio de Ambiente y Desarrollo Sostenible. Decreto 1076 de 2015. Por medio del cual se expide el
Decreto Único Reglamentario del Sector Ambiente y Desarrollo Sostenible (26 mayo 2015).

Dean, M. (2010). Governmentality. Power and rule in modern society. Londres: Sage Publications Ltd.

Deleuze, G. (1991).What is a dispositif? En T. J. Armstrong (Ed.), Michel Foucault: Philosopher (pp. 159-68). Nueva York: Harvester Wheatsheaf.

Díaz, M. (2010). Gestión socioambiental en megaproyectos, un enfoque integrador. Medellín: Fundación Codesarrollo.

Empresas Públicas de Medellín E.S.P. (EPM). (2002). Estudio de impacto ambiental del proyecto hidroeléctrico Porce III. Medellín: Centro de Información Ambiental Corantioquia.

Escobar, A. (1986). La invención del desarrollo en Colombia. Lecturas de Economía, 20, 9-35.

Escobar, A. (2007). La invención del Tercer Mundo: construcción y deconstrucción del desarrollo. Bogotá: Editorial Norma.

Escobar, A. (2012). Una minga para el postdesarrollo: lugar, medio ambiente y movilización social en las transformaciones globales. Bogotá: Ediciones desde abajo.

Ferguson, J., \& Lohmann, L. (1994). The anti-politics machine: "Development" and bureaucratic power in Lesotho. The Ecologist, 24(5), 176-181.

Foucault, M. (1980). Power/knowledge. Selected interviews and other writings 1972-1977. Nueva York: Pantheon Books. 
Foucault, M. (2008). Segurança, território, população. São Paulo: Martin Fontes.

Gómez, L. (2010). Desarrollo social con energía. La Central Hidroeléctrica Guatapé, factor de desarrollo. Medellín: Fundación Codesarrollo.

Instituto Colombiano de Normas Técnicas y Certificación. (2015). Norma Técnica Colombina NTC-ISO 14001.

Miller, P., \& Rose, N. (2012). Governando o presente: gerenciamento da vida econômica, social e pessoal. Ferreira, P. (Trad.). São Paulo: Paulus.

Muñoz, E. (2013). Mineração e regulação social na Amazônia: o caso da mineradora Alcoa e as comunidades de Juruti Velho, Pará. En H. Acselrad (Org.), Cartografia Social, terra e território (pp. 237-274). Río de Janeiro: Universidad Federal de Río de Janeiro - Instituto de Investigación y Planeación Urbana y Regional (IPPUR).

Muñoz, G. (2015). Formas de violencia en la construcción de la central hidroeléctrica Belo Monte. [In]Justicia Hídrica. Resistencias y Alternativas en América Latina, 2, 28-34.

Muñoz, G. (2016). De atingido a empreendedor: a política em tempos de "Gestão Social". O caso da Usina Hidrelétrica Porce III na Colômbia. (Tesis doctoral IPPUR, Universidad Federal, Río de Janeiro). Doi: http://dx.doi. org/10.13140/RG.2.2.27909.45280

Muñoz, G. (2017). Las técnicas de la "gestión social" de poblaciones impactadas por proyectos hidroeléctricos como instrumento de gobierno. El Ágora, 17(2), 387-412. Doi: http://dx.doi. org/10.21500/16578031.3280

Norte Energia, Central Hidroeléctrica Belo Monte. (2011). Conozca la Central Hidroeléctrica Belo Monte.

Medio Ambiente. (27 de septiembre de 2014). ¿Qué pasa con las licencias ambientales exprés? Revista Semana. Recuperado de https://www.semana.com/ nacion/articulo/que-pasa-con-las-licencias-ambientales-expres/404177-3

Rodríguez, M., \& Espinoza, G. (2002). Gestión ambiental en América Latina y el Caribe: evolución, tendencias y principales prácticas. Washington D.C: Banco Interamericano de Desarrollo.

Sleman-Chams, J., \& Velásquez-Muñoz, C. (2016). La licencia ambiental: ¿̇instrumento de comando y control por excepción? Vniversitas, 132, 483-514. Doi: http://dx.doi.org/10.11144/ Javeriana.vjl32.laic

Unidad de Planeación Minero Energética. (2013). Proyección de demanda de energía eléctrica en Colombia. Bogotá, Minesterio de Minas y Energía de la República de Colombia.

Vainer, C. (2008). Conceito de "Atingido": uma revisão do debate. En F. Rothman (Org.), Vidas Alagadas - conflitos socioambientais, licenciamento e barragens (pp. 39-63). Viçosa: UFV. territarias 41 243 
